\title{
Theory of metaparticles
}

\author{
Laurent Freidel, ${ }^{1}$ Jerzy Kowalski-Glikman, ${ }^{2,3}$ Robert G. Leigh, ${ }^{1,4}$ and Djordje Minic ${ }^{5}$ \\ ${ }^{1}$ Perimeter Institute for Theoretical Physics, 31 Caroline Street North, Waterloo Ontario N2L 2Y5, Canada \\ ${ }^{2}$ Institute for Theoretical Physics, University of Wroclaw, Place Maksa Borna 9, 50-204 Wroclaw, Poland \\ ${ }^{3}$ National Centre for Nuclear Research, Hoża 69, 00-681 Warsaw, Poland \\ ${ }^{4}$ Department of Physics, University of Illinois, 1110 West Green Street, Urbana, Illinois 61801, USA \\ ${ }^{5}$ Department of Physics, Virginia Tech, Blacksburg, Virginia 24061, USA
}

(Received 5 January 2019; published 26 March 2019)

\begin{abstract}
We introduce and develop the theory of metaparticles. At the classical level, this is a world-line theory with the usual reparametrization invariance and two additional features. The theory is motivated by string theory on compact targets and can be thought of, at least at the noninteracting level, as a theory of particles at a given string level, or as a particle model for Born geometries. The first additional feature of the model is the presence of an additional local symmetry, which from the string point of view corresponds to the completion of worldsheet diffeomorphism invariance. From the particle world-line point of view, this symmetry is associated with an additional local constraint. The second feature is the presence of a nontrivial symplectic form on the metaparticle phase space, also motivated by string theory [L. Freidel, R. G. Leigh, and D. Minic, Noncommutativity of closed string zero modes, Phys. Rev. D 96, 066003 (2017)., L. Freidel, R. G. Leigh, and D. Minic, Intrinsic non-commutativity of closed string theory, J. High Energy Phys. 09 (2017) 060.]. Because of its interpretation as a particle model on Born geometry, the spacetime on which the metaparticle propagates is ambiguous, with different choices related by what, in string theory, we would call T-duality. In this paper, we define the model and explore some of its principle classical and quantum properties, including causality and unitarity.
\end{abstract}

DOI: $10.1103 /$ PhysRevD.99.066011

\section{INTRODUCTION}

The advent of Born geometry [1,2], as describing a target geometry of string theory upon which T-duality acts as a linear symmetry [3], presents us with many deep conceptual issues. Born geometries $[3,4]$ may be viewed as the proper generalization of double field theory (see the review [5]) and generalized geometry [6] and has recently been understood in terms of globally well-defined para-Hermitian structures [7-9]. With such structure, (meta)strings propagate generally on a target space for which our usual notion of spacetime is a subspace, usually of half-dimension; under suitable circumstances, this subspace is a Lagrangian subspace with respect to a symplectic form which is part of the defining structure of a para-Hermitian geometry and whose presence and significance in string theory has only recently been realized. It is natural to wonder about the mechanisms for localization of strings (or their corresponding particlelike states) on such submanifolds.

Published by the American Physical Society under the terms of the Creative Commons Attribution 4.0 International license. Further distribution of this work must maintain attribution to the author(s) and the published article's title, journal citation, and DOI. Funded by SCOAP ${ }^{3}$.
One related, but conceptually different, motivation for the work we present here goes back to the fundamental challenge of quantum gravity: Is it possible to reconcile Lorentz invariance with the presence of a fundamental length scale? We understand that, at some level, the reconciliation of these two seemingly incompatible concepts comes about through accepting that locality itself can be observer (or probe) dependent; in other words, that locality is relative $[10,11]$. Recently, a fundamentally new model of space called modular space $[4,12]$ was proposed as a template for a space incorporating these ideas organically into its fabric. Modular spaces appear as a choice of polarization (a commutative subalgebra) of quantum Weyl algebras that do not have any classical analogue but possess a covariant built-in length scale. Since the notion of relativistic spacetime naturally leads, upon quantization, to the concept of relativistic particles and fields, it is then natural to wonder what is the proper notion of matter compatible with modular spaces. The connection between modular spaces and string theory stems from the fundamental results that the closed string theory target is intrinsically noncommutative for the compactified modes $[13,14])$.

In this paper, we begin an exploration of such matters by introducing the metaparticle theory which may be thought of as a particle model that retains the principle zero-mode 
structure of the string and, thus, propagates on a Born geometry. Alternatively, we hope to understand the metaparticle as a modular particle, the natural relativistic fundamental excitation supported by modular space. Although its origins are in string theory, it is, in fact, an attractive new model in its own right, and we take significant effort to explain some of its classical aspects and how causality and unitarity in the corresponding quantum theory are maintained, which, from several points of view, seems problematic.

A general Born geometry $\mathcal{P}$ is endowed with three geometric structures $(\eta, \omega, H)$. In this paper, we consider only the simplest "flat" Born geometry $\sim \mathbb{R}^{2 d}$ for which $(\eta, \omega, H)$ are constant in local coordinates

$$
\mathbb{X}^{A}=\left(\begin{array}{c}
x^{\mu} \\
\tilde{x}_{\mu}
\end{array}\right), \quad \mu=0,1, \ldots, d-1 .
$$

In this coordinate space, we understand the (free) classical propagation of a particle as a world-line $\gamma: \mathbb{R} \rightarrow \mathcal{P}$, that is $\gamma: \tau \rightarrow \mathbb{X}^{A}(\tau)$. Much as for an ordinary relativistic free particle propagating in spacetime, there is an equivalent phase space formulation in which reparametrization invariance of the world line is ensured by the presence of a Hamiltonian constraint, which generates a corresponding canonical transformation on the phase space variables $\mathbb{X}^{A}(\tau), \mathbb{P}_{A}(\tau)$. At first sight, such a model must surely be sick, as there are two timelike directions, $x^{0}$ and $\tilde{x}_{0}$. However, as we describe below, the metaparticle theory is endowed with a second local constraint whose origins lie in world-sheet diffeomorphism invariance of the parent string theory. This second constraint is in particular responsible for the restoration of causality and unitarity in the metaparticle theory.

The paper is organized as follows. In Sec. II, we define the metaparticle theory and present key features of its quantum propagator. In Sec. III, we revisit the notion of causality for the usual relativistic particle and present the relationship between the causality property of the metaparticle and the positivity property of the Lagrange multipliers associated with the two local constraints. We also present a decisive proof that the dispersion relations for the metaparticle do not violate unitarity. In Sec. IV, we present the symmetries of the metaparticle. We show that we have a doubling of both the Lorentz symmetry group and the world-line diffeomorphism group, establishing that metaparticles are fundamentally relativistic. In Sec. V, we present a preliminary discussion of the coupling of metaparticles to background fields.

\section{THE METAPARTICLE DEFINED}

Recall that the classical dynamics of a relativistic particle can be described in phase space coordinates $\left\{x^{\mu}(\tau), p_{\mu}(\tau)\right\}$ on a world line coordinatized by $\tau$

$$
\begin{aligned}
S & =\int d \tau\left(p_{\mu}(\tau) \dot{x}^{\mu}(\tau)-e(\tau) \mathcal{H}(\tau)\right), \\
\mathcal{H}(\tau) & =\frac{1}{2}\left(h^{\mu \nu} p_{\mu}(\tau) p_{\nu}(\tau)+m^{2}\right) .
\end{aligned}
$$

where $h_{\mu \nu}$ is a target space metric that we take to be the constant Minkowski metric. Here, $e(\tau)$ acts as a Lagrange multiplier for the Hamiltonian constraint. We can see by inspection that configurations upon which the constraint vanishes correspond to on-shell particle propagation, the momentum satisfying ${ }^{1} p^{2}=-m^{2}$. In this construction, $x^{\mu}(\tau)$ act as Lagrange multipliers forcing the conservation of $p_{\mu}(\tau)$. Passing to the Lagrangian formulation by integrating out $p_{\mu}$ through its equation of motion $\dot{x}^{\mu}(\tau)=e(\tau) h^{\mu \nu} p_{\nu}(\tau)$, yields the action

$$
S=\frac{1}{2} \int d \tau\left(\frac{1}{e(\tau)} h_{\mu \nu} \dot{x}^{\mu}(\tau) \dot{x}^{\nu}(\tau)-m^{2} e(\tau)\right)
$$

and by integrating out $e(\tau)$ the familiar coordinate space action

$$
S=-m \int d \tau \sqrt{-h_{\mu \nu} \dot{x}^{\mu}(\tau) \dot{x}^{\nu}(\tau)}
$$

in terms of the induced length of the world line in target space. The $e$ equation of motion gives ${ }^{2}$

$$
e(\tau)=\frac{1}{m} \sqrt{-h_{\mu \nu} \dot{x}^{\mu}(\tau) \dot{x}^{\nu}(\tau)}
$$

and the quantity in the square root is minus the metric on the world line induced by the embedding $\gamma: \tau \rightarrow x^{\mu}(\tau)$. Thus, we identify $e(\tau)$ with the world-line "co-frame," and onshell, the action computes the proper time along the curve, $S=-m^{2}\left(\ell_{f}-\ell_{i}\right)=-m^{2} \int d \tau e(\tau)$.

The metaparticle is defined in a doubled target phase space of Lorentzian signature, whose coordinates we label $\left\{x^{\mu}, p_{\mu}, \tilde{x}_{\mu}, \tilde{p}^{\mu}\right\}$, with $\mu=0,1, \ldots, d-1$. If we were simply to write down the ordinary particle action on this phase space, there would be a physical problem owing to the signature of the coordinate space. So, in addition to the Hamiltonian constraint, which now reads

$$
\begin{aligned}
\mathcal{H} & =\frac{1}{2}\left(p^{2}+\tilde{p}^{2}+m^{2}\right) \\
& =\frac{1}{2}\left(h^{\mu \nu} p_{\mu} p_{\nu}+h_{\mu \nu} \tilde{p}^{\mu} \tilde{p}^{\nu}+m^{2}\right),
\end{aligned}
$$

\footnotetext{
${ }^{1}$ We use 'mostly plus' signature throughout the paper.

${ }^{2}$ Note that we have been compelled to make a choice of sign for the square root, which here corresponds to assuming $e>0$. We will return later to a more complete discussion of this issue, as it is closely related to causality.
} 
we introduce a second constraint

$$
\mathcal{D}=p_{\mu} \tilde{p}^{\mu}-\mu,
$$

along with a second Lagrange multiplier that we call $\tilde{e}$. In the analogous string theory, these two constraints are associated with world-sheet diffeomorphism invariance, ${ }^{3}$ and setting them to zero on quantum states gives the onshell conditions for particle states ${ }^{4}$ whose oscillator levels are associated with values of $m^{2}$ and $\mu$. Thus, in the string theory analogue, the metaparticle theory corresponds (for specific values of $\mathrm{m}^{2}, \mu$ ) to a formulation of the dynamics of particle states at a fixed level. Later in the paper, we will address positivity requirements on $e$ and $\tilde{e}$. We will also discuss later the symmetries of the model; here we simply note that the $\mathcal{H}$-constraint is invariant under $O(2,2 d-2)$, while the $\mathcal{D}$-constraint breaks that to a subgroup which includes an apparent doubling of the Lorentz group $O(1, d-1)$.

The second ingredient in the specification of the metaparticle is the symplectic structure, which we take to be

$$
\omega=\delta p_{\mu} \wedge \delta x^{\mu}+\delta \tilde{p}^{\mu} \wedge \delta \tilde{x}_{\mu}+\pi \alpha^{\prime} \delta p_{\mu} \wedge \delta \tilde{p}^{\mu} .
$$

This form of the symplectic structure is again motivated by that of the zero modes of the Polyakov string on compact spacetime $[13,14]$. The third term, which depends on an additional parameter $\alpha^{\prime}$, leads to noncommutativity of $x^{\mu}$ and $\tilde{x}_{\mu}$, and so the phase space coordinates that we are using here are not quite Darboux coordinates. One recovers the Darboux parametrization in the limit $\alpha^{\prime} \rightarrow 0$. It may seem artificial to not simply diagonalize the symplectic form, but we choose not to do so as, in the context of string theory $x, \tilde{x}$ are preferred coordinates, and more generally we expect that the introduction of interactions will single these out as the most natural. The third term in the symplectic form has interesting consequences for the dynamics of metaparticles. ${ }^{5}$ Generally, for a function $f$ on phase space, we define the corresponding Hamiltonian vector field ${ }^{6} \xi_{f}$ via $-\omega\left(\xi_{f}, \cdot\right)=\delta f$. The Poisson bracket of functions $f$ and $g$ is given by

\footnotetext{
${ }^{3} \mathcal{H}$ and $\mathcal{D}$ are associated with world-sheet time and space reparametrizations, respectively.

${ }^{4}$ By allowing (the eigenvalues of) $\tilde{p}$ to be nonzero, we are effectively compactifying the target space. In standard notation, we have $\alpha^{\prime} m^{2} / 2=N+\tilde{N}-2$ and $\mu \alpha^{\prime}=N-\tilde{N}$. Thus, in the string interpretation, $\mathrm{m}^{2}, \mu$ are quantized in units of the string length, although here we simply take them as continuous parameters.

${ }^{5}$ It is useful to note that the Poisson brackets derived from (7) are reminiscent of the Dirac brackets found for charged particles in a magnetic field, reduced to the lowest Landau level. This observation is useful in understanding the canonical quantization of the system [12].

${ }^{6}$ When $\omega$ is closed and nondegenerate at least, this Hamiltonian vector field is unique.
}

$$
\{f, g\} \equiv \xi_{f}(g)=\delta g\left(\xi_{f}\right)=\omega\left(\xi_{f}, \xi_{g}\right)
$$

The symplectic structure (7), therefore, implies the following nontrivial equal-time brackets:

$\left\{p_{\mu}, x^{\nu}\right\}=\delta_{\mu}^{\nu}, \quad\left\{\tilde{p}_{\mu}, \tilde{x}^{\nu}\right\}=\delta_{\mu}^{\nu}, \quad\left\{\tilde{x}_{\mu}, x^{\nu}\right\}=\pi \alpha^{\prime} \delta_{\mu}^{\nu}$.

As mentioned above, we see that the effect of the $\alpha^{\prime}$ term is to render the coordinates $(x, \tilde{x})$ noncommutative. Consequently, if we were to reduce from phase space to the Born geometry $x, \tilde{x}$, the resulting theory would be nonlocal on the world line. We will not refer to this further in the present paper, in favor of discussing the theory in a particular Lagrangian subspace, which we will take to be coordinatized by $x^{\mu}, \tilde{p}^{\mu}$. In the quantum theory, we specify this as a choice of transition amplitude, related to world lines with fixed $x, \tilde{p}$ at its endpoints. As we will see, this has an interesting interpretation in which the $x^{\mu}$ coordinatize spacetime, with $\tilde{p}^{\mu}$ representing additional quantum numbers. It should be clear though that there is nothing special about the $x^{\mu}$; we could have taken any linear subspace, such as $\tilde{x}, p$. Generally, other polarizations can be obtained from our analysis by generalized Fourier transforms and will be considered elsewhere.

Let us begin by exploring some classical aspects of the metaparticle. The detailed form of the action depends on the above-mentioned choice of boundary conditions, which specifies a choice of presymplectic 1-form $\Theta$, with $\omega=\delta \Theta$. In the present case, the metaparticle action is given by

$$
S=\int \mathrm{d} \tau\left[p \cdot \dot{x}-\tilde{x} \cdot \dot{\tilde{p}}+\pi \alpha^{\prime} p \cdot \dot{\tilde{p}}-e \mathcal{H}-\tilde{e} \mathcal{D}\right]
$$

Its variation leads to the equations of motion of the form

$$
\begin{array}{lll}
e h^{\mu \nu} p_{\nu}+\tilde{e} \tilde{p}^{\mu}=\dot{x}^{\mu}+\pi \alpha^{\prime} \dot{\tilde{p}}^{\mu}, & \mathcal{H}=0, & \dot{p}_{\mu}=0 \\
e h_{\mu \nu} \tilde{p}^{\nu}+\tilde{e} p_{\mu}=\dot{\tilde{x}}_{\mu}-\pi \alpha^{\prime} \dot{p}_{\mu}, & \mathcal{D}=0, & \dot{\tilde{p}}^{\mu}=0
\end{array}
$$

Thus, we see that in the case of a free classical metaparticle, the effects of the $\alpha^{\prime}$ term drop out if we impose the $(x, \tilde{x})$ equations of motion, that force the momenta to be constant. Thus, were it not for the $\mathcal{D}$ gauge constraint, the classical motion would apparently be that of a particle in a doubled spacetime. The $\alpha^{\prime}$ term will however have a nontrivial effect in the quantum theory, especially in the presence of interactions. One of the reasons for the need for an extra constraint is that the doubling of position variables also implies a doubling of their time components. We therefore need two constraints to saturate each of the time directions and ensure that the metaparticle dynamics is causal. We will provide much more detail on this point in what follows. 


\section{A. Quantum propagator}

To better understand the metaparticle, in this subsection, we present an overview of the structure of the quantum propagator. A more complete analysis, including a discussion of choices that will be made here, appears in a later section. As we mentioned above, we will confine our attention in this paper to the $x, \tilde{p}$ polarization, which in the quantum theory means that we are considering a specific set of transition amplitudes. Since we have two constraints, the quantum states will be further labelled by a pair of evolution parameters, which we call $\ell, \tilde{\ell}$. The transition amplitude in the $x, \tilde{p}$ polarization has a canonical interpretation

$$
\begin{aligned}
& K\left(x_{f}, \tilde{p}_{f}, \ell_{f}, \tilde{\ell}_{f} ; x_{i}, \tilde{p}_{i}, \ell_{i}, \tilde{\ell}_{i}\right) \\
& \quad=\left\langle x_{f}, \tilde{p}_{f} ; \ell_{f}, \tilde{\ell}_{f} \mid x_{i}, \tilde{p}_{i} ; \ell_{i}, \tilde{\ell}_{i}\right\rangle \\
& \quad=\left\langle x_{f}, \tilde{p}_{f}\left|e^{-i\left(\ell_{f}-\ell_{i}\right) \hat{\mathcal{H}}-i\left(\tilde{\ell}_{f}-\tilde{\ell}_{i}\right) \hat{\mathcal{D}}}\right| x_{i}, \tilde{p}_{i}\right\rangle .
\end{aligned}
$$

As we will see later in more detail, causality imposes that $\ell=\ell_{f}-\ell_{i}>0$ while we will not restrict the sign of $\tilde{\ell}=\tilde{\ell}_{f}-\tilde{\ell}_{i}$. The relativistic propagator, denoted $G$, is obtained by integrating $K$ over $\ell$ and $\tilde{\ell}$. This comes about when we introduce a parameter $\tau$ parametrizing the world line and express the Hamiltonian parameters in terms of the frame fields $(e, \tilde{e})$

$$
\ell=\int_{\mathcal{C}}|e|(\tau), \quad \tilde{\ell}=\int_{\mathcal{C}} \tilde{e}(\tau) .
$$

where $\mathcal{C}: \tau \rightarrow(x, \tilde{x}, p, \tilde{p}, e, \tilde{e})(\tau)$ is a path in phase space, with boundary conditions $(x, \tilde{p})\left(\tau_{i}\right)=\left(x_{i}, \tilde{p}_{i}\right)$ and $(x, \tilde{p})\left(\tau_{f}\right)=\left(x_{f}, \tilde{p}_{f}\right)$, and the modulus on $e$ follows from the causality requirement, which will be explained in detail in the next section.

As in the usual derivation of the particle path integral, we use factorization repeatedly to find a continuum expression for G. Allowing for arbitrary parametrizations of the world line yields the integration over $e$ and $\tilde{e}$, as is explained in detail in Appendix. This in turn means that the propagator $G$ is obtained by integrating over all Lagrange parameters and is independent of the coordinate times $\tau_{i}, \tau_{f}$. Thus, we find

$$
\begin{aligned}
G\left(x_{f}, \tilde{p}_{f} ; x_{i}, \tilde{p}_{i}\right) \\
=\int[\operatorname{ded} \tilde{e}] \int_{x_{i}, \tilde{p}_{i}}^{x_{f}, \tilde{p}_{f}}\left[d^{d} x d^{d} \tilde{p}\right] \\
\quad \times \int\left[d^{d} p d^{d} \tilde{x}\right] e^{i \int_{\mathcal{C}}\left(p \cdot \mathrm{d} x-\tilde{x} \cdot \mathrm{d} \tilde{p}+\pi \alpha^{\prime} p \cdot \mathrm{d} \tilde{p}-|e| \mathcal{H}-\tilde{e} \mathcal{D}\right),}
\end{aligned}
$$

The derivation of this path integral proceeds without incident because the operators $\hat{x}, \hat{\tilde{p}}$ commute. Computing it, we find after gauge fixing, that the propagator in $x, \tilde{p}$ space is

$$
\begin{aligned}
& G\left(x_{f}, \tilde{p} ; x_{i}, \tilde{p}_{i}\right) \\
& \sim \delta^{(d)}\left(\tilde{p}-\tilde{p}_{i}\right) \int \frac{d^{d} p}{(2 \pi)^{d}} \int d \ell d \tilde{\ell} e^{-i \ell \mathcal{H}-i \tilde{\ell} \mathcal{D}} e^{i p \cdot\left(x_{f}-x_{i}\right)},
\end{aligned}
$$

Integrating over $\ell \in(0, \infty)$ and $\tilde{\ell} \in(-\infty, \infty)$, then yields the metaparticle propagator

$$
\begin{aligned}
G\left(x, \tilde{p} ; 0, \tilde{p}_{i}\right) \sim & \delta^{(d)}\left(\tilde{p}-\tilde{p}_{i}\right) \int \frac{d^{d} p}{(2 \pi)^{d}} \frac{e^{i p \cdot x}}{p^{2}+\tilde{p}^{2}+m^{2}-i \varepsilon} \\
& \times \delta(p \cdot \tilde{p}-\mu) .
\end{aligned}
$$

There are two differences compared to an ordinary relativistic particle propagator. One is the $\delta$-function of the $\mathcal{D}$ constraint, and the other is the presence of $\tilde{p}$ in the denominator. We note that the propagator is invariant under the change $\mu \rightarrow-\mu$, if we simultaneously change $\tilde{p} \rightarrow-\tilde{p}$. Consequently, we will, without loss of generality, assume that $\mu>0$.

To come to an understanding of the metaparticle propagator, suppose we take $\tilde{p}$ to be spacelike, $\tilde{p}^{\mu}=\mathcal{P} \tilde{n}^{\mu}$, where $\tilde{n}^{2}=1$, and we can parametrize $p$ as $p^{\mu}=(p \cdot \tilde{n}) \tilde{n}^{\mu}+p_{\perp}^{\mu}$ with $\tilde{n} \cdot p_{\perp}=0$ and similarly for $x$. This means in particular that $p_{\perp}$ can be timelike. The propagator (stripped of the $\delta$-function) then reads

$G(x, \mathcal{P}, \tilde{n}) \sim \int \frac{d^{d-1} p_{\perp}}{|\mathcal{P}|} \frac{e^{i\left(\frac{\mu}{\tilde{p}} \tilde{+}+p_{\perp}\right) \cdot x}}{p_{\perp}^{2}+(\mathcal{P}-\mu / \mathcal{P})^{2}+m^{2}+2 \mu-i \varepsilon}$.

We see that the effect of the $\mathcal{D}$-constraint is to effectively fix the component of the momentum parallel to $\tilde{p}{ }^{7}$ The dispersion is relativistic since there are two energy poles, albeit with a modified $\mathcal{P}$-dependent dispersion relation,

$$
p_{0}= \pm \sqrt{\vec{k}^{2}+\mathcal{P}^{2}+m^{2}+\mu^{2} / \mathcal{P}^{2}}
$$

These have a particle and antiparticle interpretation, with an effective $\mathcal{P}$-dependent pole mass.

Somewhat more difficult to interpret is the case of timelike or null $\tilde{p}$. Indeed, suppose that $\tilde{p}$ is timelike. ${ }^{8}$ We write $\tilde{p}^{\mu}=\mathcal{E} \tilde{n}^{\mu}$, where $\tilde{n}^{2}=-1$ and $\mathcal{E}$ will be referred to as the dual energy. We parametrize $p$ as $p^{\mu}=-(p \cdot \tilde{n}) \tilde{n}^{\mu}+p_{\perp}^{\mu}$

\footnotetext{
${ }^{7}$ In the string analogue, the usual interpretation of this result would be that the string state sees the dimensions transverse to $\tilde{p}$ as noncompact (or at least does not detect a compactification radius), and the dimension parallel to $\tilde{p}$ as compact. The $\mathcal{D}$ constraint determines a discrete value of the momentum given $\mu$ and $\tilde{p}$, but does not determine the compactification radius.

${ }^{8}$ In the string analogue, the only apparently available interpretation is that we are introducing winding modes in a compact Lorentzian time direction. We are certainly not advocating this interpretation here, but instead are suggesting an alternative causal and unitary interpretation.
} 
where $p_{\perp}$ is space-like and satisfies $\tilde{n} \cdot p_{\perp}=0$, and similarly for $x$. The propagator reads

$$
G(x, \mathcal{E}, \tilde{n}) \sim \int \frac{d^{d-1} p_{\perp}}{|\mathcal{E}|} \frac{e^{i\left(-\frac{\mu}{\mathcal{E}} \tilde{n}+p_{\perp}\right) \cdot x}}{-(\mathcal{E}-\mu / \mathcal{E})^{2}+p_{\perp}^{2}+m^{2}-2 \mu-i \mathcal{E}} .
$$

If one chooses the time to be $t=x \cdot \tilde{n}$, one sees that the phase is given by $\phi=E t-\vec{k} \cdot \vec{x}$ with $\vec{k}=\vec{p}_{\perp}, \vec{x} \equiv \vec{x}_{\perp}$ and the effective energy $E=\mu / \mathcal{E}$ is inversely proportional to the dual energy. Apparently then, $G(p, \mathcal{E})$ can be interpreted as a relativistic propagator at fixed energy, albeit with a modified pole structure - the $\mu$ parameter parametrizes a modification of the dispersion relation affecting the energy itself. Indeed, the modified dispersion relation reads

$$
E_{k}^{2}+\mu^{2} / E_{k}^{2}=\omega_{k}^{2}
$$

where for brevity we have introduced $\omega_{k}:=\sqrt{\vec{k}^{2}+m^{2}}$. One recovers the usual relativistic dispersion in the limit $\mu \rightarrow 0$. More generally, we have a fourth-order equation that determines the energy poles. Although this would seem to manifestly violate Lorentz invariance, this is a naive conclusion as we will discuss later. In this regard, we should also mention that unitarity seems in question here, even though the quantum theory that we have described is manifestly unitary, given the built-in factorization properties of the path integral. We will address this issue directly in a later section.

Moreover, one sees that a mass gap develops when $m^{2}<2 \mu$ since there is no static pole at real energy unless $m^{2}>2 \mu$. One can read from these relations the phase velocity $v_{\varphi}=E_{k} / k$, the group velocity $v_{g}=\partial_{k} E_{k}$ and the refractive index $n(k)=k / E_{k}$. Note that in the limit $\mathcal{E} \rightarrow 0$, the effective energy $E_{k}$ and momentum $k$ both go to infinity. This corresponds to an optical limit and one recovers, in this limit, the usual relativistic dispersion relations $v_{\varphi}=v_{g}=1$. To analyze more precisely this limit, consider the regime $\mu / \omega_{k}^{2} \ll 1$. Focusing on the positive root that generalizes the relativistic particle (a full analysis is presented later) we see that

$$
\begin{aligned}
E_{k}^{(+)} & =\frac{1}{2}\left(\sqrt{\omega_{k}^{2}+2 \mu}+\sqrt{\omega_{k}^{2}-2 \mu}\right) \\
& =\omega_{k}-\frac{1}{2} \frac{\mu^{2}}{\omega_{k}^{3}}-\frac{5}{8} \frac{\mu^{4}}{\omega_{k}^{7}}+\cdots,
\end{aligned}
$$

and so one recovers in this regime the usual dispersion relation plus corrections. In general, the group velocity differs from the phase velocity and the effective refractive index is not unity. We will discuss the propagator more completely in the following section.

Finally, when $\tilde{p}$ is null, it can be parametrized as $\tilde{p}=$ $\mathcal{E}(1, \vec{n})$ with $\vec{n}^{2}=1$, and we find that the $\delta$ function fixes the light-cone momentum. More precisely, we have $p=p_{-}(1, \vec{n})-\frac{\mu}{2 \mathcal{E}}(1,-\vec{n})+\left(0, \vec{p}_{\perp}\right)$ for any $\left(p_{-}, \vec{p}_{\perp}\right)$ with $\vec{n} \cdot \vec{p}_{\perp}=0$. In this case, we find

$G(x, \mathcal{E}, \tilde{n}) \sim \int d^{d-2} p_{\perp} \int d p_{-} \frac{e^{i\left(-\frac{\mu}{\varepsilon} x^{+}+p_{-} x^{-}+\vec{p}_{\perp} \cdot \vec{x}_{\perp}\right)}}{-2 \mu p_{-}+\mathcal{E} \vec{p}_{\perp}^{2}-i \varepsilon}$,

which contains a single nonrelativistic pole with an effective mass $\mu / \mathcal{E}$. It is possible that this is related to nonrelativistic strings [15], although we do not pursue this here. All in all, we see that the metaparticle propagator has very interesting structure that deserves further exploration.

Note that when $\mu$ is zero, it is possible to consider only external states with $\tilde{p}=0$. In this case, the $\delta$ function has no restriction on the particle momenta, and the pole is the same as in the relativistic particle. The same conclusion applies when imposing $\mu=0$ in the dispersion (46). This shows that the usual relativistic particle can be viewed as a metaparticle which is "massless" for the $\mathcal{D}$ constraint and which possesses vanishing external dual momentum. This sector corresponds to a consistent truncation of the theory since the vanishing of $\tilde{p}$ is consistent with momentum conservation and is preserved by the interactions. In the analogue string theory, this is the consistent truncation of the spectrum that occurs in the decompactification limit.

Note that if different metaparticles carry different nonzero values of $\tilde{p}$, then they have different dispersion relations and de facto effectively experience different notions of spacetime even if they have the same mass. Furthermore, since $\tilde{p}$ is conserved, the introduction of interactions implies that there must be metaparticles with distinct values of $\tilde{p}$. This is a manifestation of what has been called relative locality $[10,11]$, and it is a built-in feature of the metaparticle theory.

\section{CAUSALITY: FEYNMAN VS HADAMARD}

We now explore more completely several physical issues, including causality and unitarity. To begin, let us return briefly to the world-line formulation of ordinary particles in a spacetime $M$. As we have mentioned, the proper time is obtained by integrating $e$ along the world line. More precisely, we have two choices for the relativistic particle action. In the quantum theory, we interpret these two choices as distinct choices of the measure of the integration over $e(\tau)$, and, thus, the quantum transition amplitude computes different things in each case. The first option is to regard $\hat{e}:=e(\tau) d \tau$ as a one-form on the world line, and we take

$$
S_{H}(\mathcal{C})=-m^{2} \int_{\mathcal{C}} \hat{e}
$$

We refer to this case as the Hadamard particle.

The second option, which we will refer to as the Feynman particle (and is in fact the case considered in 
the previous section), is to consider instead the integral of the density $|\hat{e}|$ associated with $\hat{e}$ :

$$
S_{F}(\mathcal{C})=-m^{2} \int_{\mathcal{C}}|\hat{e}|
$$

Under diffeomorphisms, the coordinates transform as scalars and $\hat{e}$ as a one form:

$$
\begin{aligned}
\tau & \rightarrow \xi(\tau), \quad x^{\mu}(\tau) \rightarrow x^{\mu}(\xi(\tau)), \\
e(\tau) & \rightarrow \dot{\xi}(\tau) e(\xi(\tau)), \quad|\hat{e}|(\tau) \rightarrow|\hat{e}|(\xi(\tau)) .
\end{aligned}
$$

The Feynman action $S_{F}$ is invariant under arbitrary diffeomorphisms, including those that reverse orientation of the curve $\mathcal{C}$. The Hadamard action $S_{H}$ is on the other hand invariant under only orientation-preserving diffeomorphisms and requires the world line to be oriented. There are two critical differences between these theories, which are crucial at the quantum level. The first one is that the symmetry group for the Feynman particle is bigger than the one for the Hadamard particle by a $\mathbb{Z}_{2}$ factor. This affects the construction of the propagator. Moreover, since the Feynman particle action is always positive, it corresponds to strictly causal propagation, while the Hadamard action does not.

We see that the action (1) is the Hadamard action, while in the Feynman case, we would refine the action to the form

$$
S_{F}=\int_{\mathcal{C}} p_{\mu} \dot{x}^{\mu}-\int_{\mathcal{C}}|\hat{e}| \mathcal{H}
$$

The difference is seen in the behavior under time reversal. The Feynman action is time reversal invariant, i.e., $T S_{F}(\mathcal{C}) T^{-1}=S_{F}(-\mathcal{C})=S_{F}(\mathcal{C})$. This means that at the quantum level, time reversal is implemented as an antiunitary operator in order to satisfy $T e^{i S_{F}(\mathcal{C})} T^{-1}=\left[e^{i S_{F}(\mathcal{C})}\right]^{*}$. The Hadamard action changes sign under time reversal, $T S_{H}(\mathcal{C}) T^{-1}=S_{H}(-\mathcal{C})=-S_{H}(\mathcal{C})$ and this allows time reversal to be implemented unitarily instead. Once again the key difference is in their causality properties. We will return shortly to a similar but more involved discussion in the context of the metaparticle.

The world-line formulation of the Feynman particle in coordinate space is obtained after integrating $p$ which leads to the equation $p_{\mu}=|e| h_{\mu \nu} \dot{x}^{\nu}$. Demanding that the energy $p^{0}$ is positive solders the orientation of the target time $x^{0}$ to the orientation of the world line. To understand this fact let's first appreciate that the particle possesses in principle two notions of time orientation. We have the world-line orientation that tracks the flow of proper-time $\tau$ and the target time orientation that tracks the motion of $x^{0}$. So we are at risk to have two notions of the future, the one measured by world-line clocks and the one measured by observers in spacetime.
The Feynman condition eliminates this possibility by ensuring that positive energy particles are exactly the particles that have identical notions of world-line and target causality. Moreover, under a diffeomorphism that changes the orientation of the world-line $(e, \tau) \rightarrow(-e,-\tau)$ and the target time orientation $x^{0} \rightarrow-x^{0}$, the timelike component of the velocity and the energy are unchanged so that a particle is mapped onto an antiparticle. It is in this sense that an antiparticle can be understood as a particle moving backward in time as first understood by Stueckelberg [16]. Therefore a nonorientable trajectory (from the world-line point of view) corresponds to a collection of pair creations or annihilations.

In the Hadamard version of the theory, we have instead that $p^{\mu}=e \dot{x}^{\mu}$ and depending on the sign of $e$, a positive energy particle does not necessarily correspond to a particle having matching world-line and target time orientability. It is instead the sign of $e$ that controls these features. Thus, if we demand that admissible Hadamard particles have matching time orientation we are effectively considering world lines that are positively oriented $e>0$, while the energy can be negative or positive. The first condition means that we cannot create a loop and the second that we would see negative energy excitations. This clearly rules out this as a possible description of physical particles.

The path integral has a canonical interpretation as a quantum transition matrix element. In order to perform this integral, one has to divide out by the gauge symmetry acting on $e$. For example, if we take $\Theta=p_{\mu} \delta x^{\mu}$ and fix the field configurations $x^{\mu}\left(\tau_{i, f}\right)=x_{i, f}^{\mu}$ with the world line parametrized by $\tau \in\left[\tau_{i}, \tau_{f}\right]$, we have

$\left.\left\langle x_{f} \mid x_{i}\right\rangle \sim \int[D e(\tau)]\left[D x^{\mu}(\tau) D p_{\mu}(\tau)\right]\right|_{x_{i}} ^{x_{f}} e^{i \int_{\mathcal{C}}\left(p_{\mu} d x^{\mu}-|\hat{e}| \mathcal{H}\right)}$.

Alternatively, we could specify the momentum at the endpoints of the world line, obtaining

$\left.\left\langle p_{f} \mid p_{i}\right\rangle \sim \int[D e(\tau)]\left[D x^{\mu}(\tau) D p_{\mu}(\tau)\right]\right|_{p_{i}} ^{p_{f}} e^{i \int_{\mathcal{C}}\left(-x^{\mu} d p_{\mu}-|\hat{e}| \mathcal{H}\right)}$.

These, of course, are related by Fourier transform. Now, what exactly these path integrals compute depends on the details of the integral over the form $e$. This is easiest to understand given the form (29). In that case, the free integral over the $x^{\mu}(\tau)$ localizes the $p$-integrals on configurations with $\dot{p}_{\mu}=0$. That is, the path integral vanishes unless $p_{f}=p_{i}$, and

$\left\langle p_{f} \mid p_{i}\right\rangle \sim \delta^{(d)}\left(p_{f}-p_{i}\right) \int[D e(\tau)] e^{-i \frac{1}{2}\left(p_{f}^{2}+m^{2}\right) \int_{\mathcal{C}}|\hat{e}|}$. 
Thus, all but the zero mode $\ell=\int_{\mathcal{C}}|e|$ for Feynman and $\ell=\int_{\mathcal{C}} e$ for Hadamard decouples. Given the local symmetry (26), the nonzero modes are pure gauge and so can be discarded from the path integral. Thus,

$$
\left\langle p_{f} \mid p_{i}\right\rangle \sim \delta^{(d)}\left(p_{f}-p_{i}\right) \int d \ell e^{-i \frac{1}{2}\left(p_{f}^{2}+m^{2}\right) \ell}
$$

For the Hadamard particle, one integrates over $\ell \in(-\infty, \infty)$, and we obtain

$$
\left\langle p_{f} \mid p_{i}\right\rangle \sim \delta^{(d)}\left(p_{f}-p_{i}\right) \delta\left(p_{f}^{2}+m^{2}\right) .
$$

That is, in this case the path integral vanishes unless the states are physical, satisfying the gauge constraint $p^{2}+m^{2}=0$ (which coincides with the classical on-shell condition).

On the other hand in the Feynman case, there is an extra $\mathbb{Z}_{2}$ symmetry that makes $\ell$ positive and after integrating over $\ell \in(0, \infty)$, we obtain the causal (Feynman) propagator

$$
\left\langle p_{f} \mid p_{i}\right\rangle \sim \delta^{(d)}\left(p_{f}-p_{i}\right) \frac{1}{p_{f}^{2}+m^{2}-i \varepsilon},
$$

where we have included the convergence factor $\varepsilon$, necessary for the convergence of the $\ell$ integral. In this case, we must interpret the external states as off-shell unphysical.

If we wish to think of the particle as living in spacetime (coordinatized by $x^{\mu}$ ) rather than phase space, we could, of course, reduce the (Gaussian) path integral (28) by integrating freely over the momenta, which leads to the usual second-order form of the path integral, with Dirichlet boundary conditions. In this case, the gauge fixing, although standard, requires more elaborate methods. Alternatively, we can simply take the above results and perform the Fourier transform

$$
\begin{aligned}
\left\langle x_{f} \mid x_{i}\right\rangle & \sim \int d^{d} p_{f} d^{d} p_{i} e^{i p_{f} \cdot x_{f}+i p_{i} \cdot x_{i}}\left\langle p_{f} \mid p_{i}\right\rangle \\
& \sim \int d^{d} p e^{i p \cdot\left(x_{f}-x_{i}\right)}\left\{\begin{array}{ll}
\delta\left(p^{2}+m^{2}\right), & \text { Hadamard } \\
\frac{1}{p^{2}+m^{2}-i \varepsilon}, & \text { Feynman }
\end{array} .\right.
\end{aligned}
$$

\section{A. Metaparticle causality}

Let us now return to the metaparticle. As we have discussed, we have to deal with two local symmetries, generated by the two constraints $\mathcal{H}$ and $\mathcal{D}$, with two associated Lagrange multipliers $e$ and $\tilde{e}$, and consequently the relationship between causality on the world line and in phase space is more subtle. At the quantum level, we have a priori a fourfold ambiguity due to the choice of Hadamard or Feynman conditions for each multiplier. We define the metaparticle theory as corresponding to the Feynman choice for $e$ and the Hadamard choice for $\tilde{e}$. This is consistent with the fact that $\mathcal{H}$ generates the time evolution while $\mathcal{D}$ generates an internal symmetry (space reparametrization in the string analogue). This means that the metaparticle Hamiltonian is given by

$$
H=|e| \mathcal{H}+\tilde{e} \mathcal{D} .
$$

In Appendix, we have given a careful derivation of the discretized path integral, in the course of which it is clear that the Lagrange multiplier $\tilde{e}$ can be integrated over all real values, consistent with our choice of Hadamard for $\tilde{e}$.

To appreciate the importance of the causal $|e|$ prescription, notice that when no external fields are involved, it is possible to kinematically decouple the metaparticle system as a sum of two free relativistic particles. To do so, one introduces chiral momenta $p_{\mu}^{ \pm}=\frac{1}{2}\left(p_{\mu} \pm h_{\mu \nu} \tilde{p}^{\nu}\right)$. The total Hamiltonian can be written as the sum $H=e_{+} \mathcal{H}_{+}+e_{-} \mathcal{H}_{-}$, where we define $e_{ \pm}=e \pm \tilde{e}$ and $^{9}$

$\mathcal{H}_{ \pm}:=\left(p_{\mu}^{ \pm} h^{\mu \nu} p_{\nu}^{ \pm}+m_{ \pm}^{2}\right), \quad m_{ \pm}^{2}=\frac{1}{4}\left(m^{2} \mp 2 \mu\right)$.

The symplectic potential contains a coupling of the two chiral components through the $\alpha^{\prime}$ term. Written like this, it is clear that the metaparticle prescription, (i.e., Feynman for $\mathcal{H})$ is fundamentally different from the prescription that would treat the two chiral particles as independent (that is, the Feynman prescription for each chiral $\left|e_{ \pm}\right|$). This means that the metaparticle prescription $|e|$ creates a fundamental "entanglement" of these chiral particles which prevents an interpretation as two particle species. Remarkably, this is very similar to the description of spin given as an entangled biparticle model in $[17,18]$.

To better understand this prescription, we perform an analysis of the action similar to the analysis done for the particle in Sec. II. We continue here the analysis in the polarization in which $(x, \tilde{p})$ are the configuration space variables, leaving other choices [such as $(x, \tilde{x})$ ] to a future publication. The metaparticle action for this polarization is given by

$$
S=\int \mathrm{d} \tau\left[p \cdot \dot{x}-\tilde{x} \cdot \dot{\tilde{p}}+\pi \alpha^{\prime} p \cdot \dot{\tilde{p}}-|e| \mathcal{H}-\tilde{e} \mathcal{D}\right] .
$$

One can integrate the corresponding "momenta" $(p, \tilde{x})$ which leads to the equations

$$
|e| p^{\mu}=(\dot{x}-\tilde{e} \tilde{p})^{\mu}, \quad \dot{\tilde{p}}^{\mu}=0 .
$$

\footnotetext{
${ }^{9}$ We should remark here that although one might have assumed by notation that $m^{2}$ is meant to be positive, it is not clear that $m_{ \pm}^{2}$ should be taken to be both positive. In the string analogue, we are considering here the left- and right-movers, and in standard notation we have $\alpha^{\prime} m^{2} / 2=N+\tilde{N}-2$ and $\mu \alpha^{\prime}=N-\tilde{N}$. Thus, $\alpha^{\prime} m_{+}^{2}=\tilde{N}-1$ and $\alpha^{\prime} m_{-}^{2}=N-1$.
} 
Using these to eliminate $p, \tilde{x}$ results in the configuration space action

$S=\frac{1}{2} \int \mathrm{d} \tau\left[\frac{1}{|e|}(\dot{x}-\tilde{e} \tilde{p})^{2}-|e|\left(\tilde{p}^{2}+m^{2}\right)+2 \tilde{e} \mu\right]$

which is the analogue of eq. (2). The frames $(e, \tilde{e})$ can be integrated out and expressed as functionals $[e(x, \tilde{p}), \tilde{e}(x, \tilde{p})]$. After integration, the action in terms of these functionals simply reads

$$
S=\int \mathrm{d} \tau\left[-\left(\tilde{p}^{2}+m^{2}\right)|e(x, \tilde{p})|+2 \mu \tilde{e}(x, \tilde{p})\right]
$$

which upon substitution of $e(x, \tilde{p})$ and $\tilde{e}(x, \tilde{p})$ is the analogue of eq. (3). Once again we see that the causality requirement of $|e|$ being positive is paired with the positivity of (an effective) mass squared, while the sign of $\tilde{e}$ and $\mu$ are both unrestricted.

In order to get the explicit expressions of the frames in terms of the configuration space variables, we need to resolve the $e, \tilde{e}$ equations of motion

$e^{2}\left(m^{2}+\tilde{p}^{2}\right)=-(\dot{x}-\tilde{e} \tilde{p})^{2}, \quad|e| \mu=\tilde{p} \cdot(\dot{x}-\tilde{e} \tilde{p})$.

When $\tilde{p}^{2} \neq 0$ and $\mu>0$, one finds that

$$
\begin{aligned}
|e| & =\sqrt{\frac{(\tilde{p} \cdot \dot{x})^{2}-\tilde{p}^{2} \dot{x}^{2}}{\mu^{2}+m^{2} \tilde{p}^{2}+\tilde{p}^{4}}}, \\
\tilde{e} & =\frac{\tilde{p} \cdot \dot{x}}{\tilde{p}^{2}}-\frac{\mu}{\tilde{p}^{2}} \sqrt{\frac{(\tilde{p} \cdot \dot{x})^{2}-\tilde{p}^{2} \dot{x}^{2}}{\mu^{2}+m^{2} \tilde{p}^{2}+\tilde{p}^{4}}} .
\end{aligned}
$$

One sees that the choice of sign of the square root is correlated with the positivity of the frame $e$, but does not fix the sign of the dual frame, consistent with our choices described above. Also one sees that $|e|$ is a measure of proper distance in the hyperplane perpendicular to $\tilde{p}$, while $\tilde{e}$ also contains a measure of the distance along $\tilde{p}$. As we saw in Sec. II A, this is most simply interpreted in the case where $\tilde{p}$ is spacelike, and there is a simple familiar classical interpretation. More careful analysis is required for timelike $\tilde{p}$, as we saw for the propagator above. The relativistic distance can be expressed as

$$
-\dot{x}^{2}=m^{2} e^{2}+\tilde{p}^{2}\left(e^{2}-\tilde{e}^{2}\right)-2 \mu \tilde{e}|e| .
$$

For completeness we mention that when $\tilde{p}^{2}=0$, one finds that the solutions of eqs. (41) are

$$
|e|=\frac{\tilde{p} \cdot \dot{x}}{\mu}, \quad \tilde{e}=\frac{\dot{x}^{2}}{2 \mu|e|}+\frac{m^{2}|e|}{2 \mu},
$$

which also can be obtained from eqs. (42) by taking $\tilde{p}^{2} \rightarrow 0$. In this case, $|e|$ is a measure of the time density while $\tilde{e}$ is the measure of a nonrelativistic energy density, where $\mu$ plays the role of a nonrelativistic mass, while $\mathrm{m}^{2} / \mu$ plays the role of a nonrelativistic rest energy.

\section{B. Metaparticle unitarity}

We now analyse the question of unitarity. As we have seen earlier in eq. (17), when we work in the $(x, \tilde{p})$ polarization with timelike $\tilde{p}$, the metaparticle propagator becomes effectively a fixed energy propagator

$$
\begin{aligned}
G\left(x_{f}, \tilde{p}_{f} ; x_{i}, \tilde{p}_{i}\right) \sim & \delta^{(d)}\left(\tilde{p}_{f}-\tilde{p}_{i}\right) \int d^{d-1} k \frac{|E|}{\mu} \\
& \times \frac{e^{-i\left(E\left(t_{f}-t_{i}\right)-\vec{k} \cdot\left(\vec{x}_{f}-\vec{x}_{i}\right)\right)}}{(E+\mu / E)^{2}-\left(\vec{k}^{2}+m^{2}+2 \mu\right)+i \epsilon},
\end{aligned}
$$

where we have denoted $\tilde{p}^{2}=-\frac{\mu^{2}}{E^{2}}, t=\frac{E}{\mu}(\tilde{p} \cdot x)$ and $(\vec{k}, \vec{x})=$ $\left(\vec{p}_{\perp}, \vec{x}_{\perp}\right)$. In other words, the dispersion relation is

$$
(E \pm \mu / E)^{2}=\omega_{k}^{2} \pm 2 \mu .
$$

One may worry that the dispersion relation is in fact quartic and not simply quadratic, revealing a potential issue with causality and unitarity. There are indeed four real roots when ${ }^{10} \omega_{k}^{2}>2 \mu$. This is always satisfied for ${ }^{11} m^{2}>2 \mu$, while a gap emerges for $m^{2}<2 \mu$ with no real solutions for $\vec{k}^{2}<2 \mu-m^{2}$. Assuming that the reality condition $\omega_{k}^{2}>2 \mu$ is satisfied, the two positive roots are

$$
\Omega_{ \pm}(k)=\frac{1}{2} \sqrt{\omega_{k}^{2}+2 \mu} \pm \frac{1}{2} \sqrt{\omega_{k}^{2}-2 \mu},
$$

while the negative roots are simply $-\Omega_{ \pm}(k)$. Two facts that will become relevant soon are that

$$
\Omega_{+} \Omega_{-}=\mu, \quad \Omega_{+}>\sqrt{\mu}, \quad \Omega_{-}<\sqrt{\mu} .
$$

As is well established [19], the condition of unitarity requires that the value of the measure $\frac{\mathrm{d} E}{\mathrm{~d} k}$ multiplied by the residue of the propagator is positive for each pole. This is due to the fact that the density $\mathrm{d} E G_{E}$, where $G_{E}$ is the propagator at fixed energy, should be interpretable as a probability distribution. This distribution localizes on the poles as $\partial_{k} \Omega(k) G_{\Omega(k)}$ and can be interpreted as a probability distribution when it is positive.

In the usual relativistic case, this is true due to the fact that the group velocity is inversely proportional to the phase velocity:

\footnotetext{
${ }^{10}$ Recall that we have chosen $\mu>0$.

${ }^{11}$ In the string analogy, this condition is implied by the demand that the oscillator numbers are greater than one $N, \tilde{N} \geq 1$, that there are no tachyonic left- or right-movers.
} 


$$
\operatorname{Res}_{ \pm \omega_{k}}\left(\frac{\partial_{k} E}{E^{2}-\omega_{k}^{2}}\right)= \pm \frac{\left.\partial_{k} E\right|_{ \pm \omega_{k}}}{2 \omega_{k}}=\frac{k}{2 \omega_{k}^{2}}>0
$$

Similarly, we can prove that this unitarity condition is satisfied by the metaparticle propagator

$$
\begin{aligned}
& \operatorname{Res}_{ \pm \Omega_{\epsilon}(k)}\left(\frac{\partial_{k} E}{(E+\mu / E)^{2}-\omega_{k}^{2}-2 \mu}\right) \\
& =\frac{ \pm\left.\epsilon \Omega_{\epsilon} \partial_{k} E\right|_{ \pm \Omega_{\epsilon}}}{2\left(\Omega_{+}^{2}-\Omega_{-}^{2}\right)}=\frac{k \Omega_{\epsilon}^{2}}{2\left(\Omega_{+}^{2}-\Omega_{-}^{2}\right)^{2}}>0
\end{aligned}
$$

where $\epsilon= \pm 1$.

This establishes the unitarity of our prescription. It is quite remarkable that all the factors conspire to give the right sign overall. One of the fundamental reasons why unitarity is respected is the fact that the two positive roots are exchanged by the duality $\Omega_{ \pm}=\mu / \Omega_{\mp}$. This duality corresponds to the exchange of $p$ with $\tilde{p}$. And under duality the sign of the propagator pole and the sign of the group velocity $\partial_{k} E$ change, so the sign of the product is unmodified. As a summary we see that we have two duality symmetries of the spectrum

$$
E \leftrightarrow-E, \quad E \leftrightarrow \frac{\mu}{E} .
$$

Both symmetries change the sign of the propagator residue and the sign of the measure Jacobian $\partial_{k} E$. The first inversion means that associated to each particle there is a corresponding antiparticle, the second inversion likewise means that, associated with a particl-antiparticle pair, there is a corresponding dual particle, dual antiparticle pair.

\section{SYMMETRIES OF THE METAPARTICLE THEORY}

Having seen the relevance of discrete symmetries for unitarity, let us now discuss more fully the global and discrete symmetries of the metaparticle action. We follow here the discussion in [3]. Let's recall that the constraints have the form

$$
\mathcal{H}=\frac{1}{2}\left(p_{\mu} h^{\mu \nu} p_{\nu}+\tilde{p}^{\mu} h_{\mu \nu} \tilde{p}^{\nu}+m^{2}\right), \quad \mathcal{D}=p_{\mu} \tilde{p}^{\mu}-\mu
$$

$\mathcal{H}$ can be written in terms of a metric $H=\operatorname{diag}\left(h, h^{-1}\right)$ which has signature $[2,2(d-1)]$ and $\mathcal{D}$ in terms of a metric of signature $(d, d)$. Let us first note that there exists a discrete $\mathbb{Z}_{2}$ duality transformation

$$
K:(\tilde{p}, p) \rightarrow(-\tilde{p}, p)
$$

which leaves invariant $\mathcal{H}$ and exchanges $\mathcal{D}_{\mu} \rightarrow \mathcal{D}_{-\mu}$. As we have seen the change $\mu \rightarrow-\mu$ is a symmetry of the spectrum and $K$ can therefore be understood as a duality ${ }^{12}$ symmetry.

The group of symmetries that preserves both Hamiltonians is $O(1, d-1) \ltimes O(1, d-1)$. The first $O(1, d-1)$ component acts diagonally

$$
R_{\Lambda}:\left(\tilde{p}^{\mu}, p_{\mu}\right) \rightarrow\left(\Lambda \tilde{p}, h \Lambda h^{-1} p\right), \quad \Lambda^{T} h \Lambda=h .
$$

The other $O(1, d-1)$ component acts as

$\tilde{R}_{\Lambda}\left(\begin{array}{c}\tilde{p} \\ p\end{array}\right)=\frac{1}{2}\left(\begin{array}{cc}(1+\Lambda) & (1-\Lambda) h^{-1} \\ h(1-\Lambda) & h(1+\Lambda) h^{-1}\end{array}\right)\left(\begin{array}{l}\tilde{p} \\ p\end{array}\right)$.

These two actions satisfy the relations

$R_{\Lambda_{1}} R_{\Lambda_{2}}=R_{\Lambda_{1} \Lambda_{2}}, \quad R_{\Lambda_{1}} \tilde{R}_{\Lambda_{2}}=\tilde{R}_{\Lambda_{1} \Lambda_{2} \Lambda_{1}^{-1}} R_{\Lambda_{1}}$

$\tilde{R}_{\Lambda_{1}} \tilde{R}_{\Lambda_{2}}=\tilde{R}_{\Lambda_{1} \Lambda_{2}}$

A special element of this symmetry group is the T-duality transformation $J:=\tilde{R}_{-1}$, which generates, together with the inversion -1 , the center of the symmetry group. It is explicitly given by

$$
J=\left(\begin{array}{cc}
0 & h^{\mu \nu} \\
h_{\mu \nu} & 0
\end{array}\right):\left(\begin{array}{c}
\tilde{p}^{\mu} \\
p_{\mu}
\end{array}\right) \mapsto\left(\begin{array}{c}
p^{\mu} \\
\tilde{p}_{\mu}
\end{array}\right), \quad J^{2}=1 .
$$

It is well known that $O(1, d-1)$ possesses four connected components interchanged by the discrete operations (1, $T, P, P T)$. Accordingly, we have two different notions of time reversal $R_{T}$ and $\tilde{R}_{T} \cdot R_{T}$ acts on both timelike components

$$
R_{T}:\left(\begin{array}{c}
x^{0} \\
\tilde{x}_{0}
\end{array}\right) \mapsto\left(\begin{array}{cc}
-1 & 0 \\
0 & -1
\end{array}\right)\left(\begin{array}{l}
x^{0} \\
\tilde{x}_{0}
\end{array}\right)
$$

which we expect to be implemented by an antilinear operator at the quantum level. The T-duality operation $J$ on the other hand is implemented unitarily.

There are additionally local symmetries. As in the previous section, let us start with the discussion of symmetries of the ordinary relativistic particle. The first order relativistic particle Lagrangian

$$
L=p \cdot \dot{x}-\frac{1}{2} e\left(p^{2}+m^{2}\right)
$$

possesses a canonical phase space gauge symmetry

$\delta_{N} x^{\mu}(\tau)=N(\tau) p^{\mu}(\tau), \quad \delta_{N} p_{\mu}(\tau)=0, \quad \delta_{N} e=\dot{N}(\tau)$

\footnotetext{
${ }^{12}$ In the string analogy, this transformation corresponds to the exchange of left and right movers.
} 
with the Lagrangian transforming by a total derivative, $L \rightarrow L+\frac{1}{2} \frac{d}{d \tau}\left(N\left(p^{2}-m^{2}\right)\right)$. This symmetry can be understood to be a canonical symmetry. In order to do so, we extend the symplectic structure of the theory by introducing $\pi$, the momentum conjugate to $e$. Let us recall that the particle action is given by

$$
S=\int\left(I_{\partial_{\tau}} \theta-H\right) \mathrm{d} \tau,
$$

where $\theta=p_{\mu} \delta x^{\mu}$ is the symplectic potential and $I_{\partial_{\tau}}$ denotes the contraction with the time flow vector. It is clear from this expression that one can add a canonical pair extending the symplectic potential $\theta_{\mathrm{ext}}=p_{\mu} \delta x^{\mu}+\pi \delta e$, and at the same time modify the hamiltonian $H_{\mathrm{ext}}=H+\pi \dot{e}$ in a manner that leaves the action unchanged. Using this extension of the symplectic structure we can now see that (60) is a canonical symmetry generated by the charge associated with the constraint

$$
H_{N}=\int\left(\frac{1}{2} N\left(p^{2}+m^{2}\right)+\dot{N} \pi\right) .
$$

It is also possible to understand the introduction of $\pi$ as the Lagrange parameter imposing a gauge condition $\dot{e}=T$, where $T(p, x)$ is a gauge fixing functional.

In order to understand the gauge fixed action, we need to extend the phase space to include also the ghost and antighost sectors (see [20] for more details). We add to the extended symplectic potential the ghost potential $\theta_{\mathrm{gh}}:=$ $b \delta c+\bar{b} \delta \bar{c}$ where the ghost variables are fermionic. The full set of conjugate pairs are

$$
\left\{p_{a}, x^{b}\right\}=\delta_{a}^{b}, \quad\{\pi, e\}=\{c, b\}=\{\bar{c}, \bar{b}\}=1 .
$$

The Hamiltonian of the gauge fixed theory can be simply described as the graded Poisson bracket $\mathcal{H}=\left\{\Theta, \Theta^{\prime}\right\}$, of two fermionic elements

$$
\Theta:=c H+\bar{b} \pi, \quad \bar{\Theta}:=\bar{c} T+b e,
$$

that are both nilpotent, $\{\Theta, \Theta\}=0=\{\bar{\Theta}, \bar{\Theta}\}$. The gaugefixed action has the form

$$
S_{\mathrm{gf}}=\int I_{\partial_{\tau}}\left(\theta_{\mathrm{ext}}+\theta_{\mathrm{gh}}\right)-\{\Theta, \bar{\Theta}\}
$$

The gauge-fixed Hamiltonian $\mathcal{H}=e H+\pi T+\bar{b} b+$ $c\{H, T\} \bar{c}$ is obviously invariant under the BRST transformation $Q \mathcal{H}=\{\Theta, \mathcal{H}\}$ since $\Theta$ is nilpotent and $\mathcal{H}=\{\Theta, \bar{\Theta}\}$. Moreover, since this is a canonical transformation, the symplectic potential, and hence the action, transforms by a total time derivative. The pair $(c, \bar{c})$ are the ghost and antighost, respectively, and the pair $(b, \bar{b})$ are their fermionic conjugates. The bosonic part of the action is the usual relativistic action plus a gauge fixing term $\pi(\dot{e}-T)$, where $\pi$ appears as a Lagrange multiplier, as promised.

The action is also invariant under the world-line diffeomorphism symmetry

$$
\begin{aligned}
\delta_{\epsilon}^{\prime} x^{\mu}(\tau) & =\epsilon(\tau) \dot{x}^{\mu}(\tau), \quad \delta_{\epsilon}^{\prime} p_{\mu}(\tau)=\epsilon(\tau) \dot{p}_{\mu}(\tau), \\
\delta_{\epsilon}^{\prime} e(\tau) & =\frac{d}{d \tau}(\epsilon(\tau) e(\tau)) .
\end{aligned}
$$

However, this symmetry differs from the canonical symmetry (60) by a trivial symmetry. ${ }^{13}$ Indeed, if we consider the difference $\Delta_{\epsilon}=\delta_{\epsilon}^{\prime}-\delta_{N=\epsilon e}$, we find that this is a trivial symmetry:

$$
\begin{aligned}
\Delta_{\epsilon} x^{\mu} & =\epsilon\left(\dot{x}^{\mu}-e p^{\mu}\right)=\epsilon \frac{\delta L}{\delta p_{\mu}}, \quad \Delta_{\epsilon} p_{\mu}=\epsilon \dot{p}_{\mu}=-\epsilon \frac{\delta L}{\delta x^{\mu}}, \\
\Delta_{\epsilon} e & =0 .
\end{aligned}
$$

This shows that the reparametrization symmetry is a combination of a Hamiltonian symmetry and a trivial symmetry. This is responsible for the fact that when the theory is reduced to coordinate space, the remaining symmetry is the diffeomorphism invariance of the coordinate space curve.

We recall that the metaparticle Lagrangian is given by

$$
L:=\left[p \cdot \dot{x}+\tilde{p} \cdot \dot{\tilde{x}}+\pi \alpha^{\prime} p \cdot \dot{\tilde{p}}-e \mathcal{H}-\tilde{e} \mathcal{D}\right]
$$

Since we have two constraints, there are two canonical symmetries of the form

$\delta_{(\alpha, \tilde{\alpha})} x=\alpha p+\tilde{\alpha} \tilde{p}, \quad \delta_{(\alpha, \tilde{\alpha})} \tilde{x}=\alpha \tilde{p}+\tilde{\alpha} p$,

$\delta_{(\alpha, \tilde{\alpha})} p=\delta_{(\alpha, \tilde{\alpha})} \tilde{p}=0, \quad \delta_{(\alpha, \tilde{\alpha})} e=\dot{\alpha}, \quad \delta_{(\alpha, \tilde{\alpha})} \tilde{e}=\dot{\tilde{\alpha}}$.

These are generated by the charges

$\mathcal{H}_{\alpha}=\int(\alpha \mathcal{H}+\dot{\alpha} \pi) d \tau, \quad \mathcal{H}_{\tilde{\alpha}}=\int(\tilde{\alpha} \mathcal{D}+\dot{\tilde{\alpha}} \tilde{\pi}) d \tau$.

where $(\pi, \tilde{\pi})$ are variables conjugate to $(e, \tilde{e})$. The action is also invariant under the world-line diffeomorphism symmetry generated by the Lie derivative $L_{\epsilon}$

$$
\begin{aligned}
& L_{\epsilon} x^{\mu}=\epsilon \dot{x}^{\mu}, \quad L_{\epsilon} \tilde{x}=\epsilon \dot{\tilde{x}}^{\mu} \quad L_{\epsilon} p_{\mu}=\epsilon \dot{p}_{\mu}, \\
& L_{\epsilon} \tilde{p}_{\mu}=\epsilon \dot{\tilde{p}}_{\mu}, \quad L_{\epsilon} e=\partial_{\tau}(\epsilon e), \quad L_{\epsilon} \tilde{e}=\partial_{\tau}(\epsilon \tilde{e}) .
\end{aligned}
$$

\footnotetext{
${ }^{13}$ A trivial symmetry of an action $S\left(\varphi^{a}\right)$ is a transformation of the form $\Delta_{\epsilon} \varphi^{a}=\epsilon^{a b} E_{b}$ where $E_{b}=\frac{\delta L}{\delta \varphi^{b}}-\partial_{\mu} P_{a}^{\mu}$ with $P_{a}^{\mu}=$ $\left(\frac{\delta L}{\delta \partial_{\mu} \varphi^{a}}\right)$, are the equations of motion and $\epsilon^{a b}=-\epsilon^{b a}$ is an arbitrary skew-symmetric tensor. The action is unchanged by such transformations while the Noether current for such a trivial symmetry is $J^{\mu}=P_{a}^{\mu} \epsilon^{a b} E_{b}$. This current vanishes on-shell.
} 
Fortunately, we can show that this symmetry differs from the canonical symmetry (69) by a trivial symmetry. To see this, consider the transformations

$$
\begin{array}{ll}
\Delta_{\epsilon} x=\epsilon(\dot{x}-e p-\tilde{e} \tilde{p}), & \Delta_{\epsilon} \tilde{x}=\epsilon(\dot{\tilde{x}}-e \tilde{p}-\tilde{e} p), \\
\Delta_{\epsilon} p=\epsilon \dot{p}, \quad \Delta_{\epsilon} \tilde{p}=\epsilon \dot{\tilde{p}}, \quad \Delta_{\epsilon} e=\Delta_{\epsilon} \tilde{e}=0 .
\end{array}
$$

On the one hand, this is a trivial symmetry since

$$
\begin{aligned}
& \Delta_{\epsilon} x=\epsilon\left(\frac{\delta L}{\delta p}+\pi \alpha^{\prime} \frac{\delta L}{\delta \tilde{x}}\right), \quad \Delta_{\epsilon} \tilde{x}=\epsilon\left(\frac{\delta L}{\delta \tilde{p}}-\pi \alpha^{\prime} \frac{\delta L}{\delta \tilde{x}}\right), \\
& \Delta_{\epsilon} p=-\epsilon \frac{\delta L}{\delta x}, \quad \Delta_{\epsilon} \tilde{p}=-\epsilon \frac{\delta L}{\delta \tilde{x}} .
\end{aligned}
$$

On the other hand, it represents the difference between a world-line diffeomorphism and a canonical symmetry with parameter $(\alpha, \tilde{\alpha})=(\epsilon e, \epsilon \tilde{e})$

$$
\Delta_{\epsilon}=L_{\epsilon}-\delta_{(\epsilon e, \epsilon \tilde{e})} .
$$

Interestingly, the action is also invariant under a T-dual version of reparametrization invariance

$$
\begin{aligned}
& \tilde{L}_{\tilde{\epsilon}} x^{\mu}=\tilde{\epsilon} \dot{\tilde{x}}^{\mu}, \quad \tilde{L}_{\tilde{\epsilon}} \tilde{x}_{\mu}=\epsilon \dot{\tilde{x}}^{\mu} \quad \tilde{L}_{\tilde{\epsilon}} p_{\mu}=\tilde{\epsilon} \dot{\tilde{p}}_{\mu}, \\
& \tilde{L}_{\tilde{\epsilon}} \tilde{p}_{\mu}=\tilde{\epsilon} \dot{p}_{\mu}, \quad \tilde{L}_{\tilde{\epsilon}} e=\partial_{\tau}(\tilde{e} \tilde{e}), \quad \tilde{L}_{\epsilon} \tilde{e}=\partial_{\tau}(\tilde{\epsilon} e) .
\end{aligned}
$$

This symmetry is just the composition of the usual reparametrization with T-duality $\tilde{L}_{\tilde{\epsilon}}=L_{\tilde{\epsilon}} J$. Interestingly, this combination can be expressed as a canonical symmetry where the role of $\alpha$ and $\tilde{\alpha}$ is interchanged. In other words, the transformation $\Delta_{\tilde{\epsilon}}=L_{\tilde{\epsilon}} J-\delta_{(\tilde{\varepsilon} \tilde{e}, \tilde{\epsilon} e)}$ given by

$$
\begin{array}{ll}
\Delta_{\tilde{\epsilon}} x=\tilde{\epsilon}(\dot{\tilde{x}}-e \tilde{p}-\tilde{e} p), & \Delta_{\tilde{\epsilon}} \tilde{x}=\tilde{\epsilon}(\dot{x}-e p-\tilde{e} \tilde{p}), \\
\Delta_{\tilde{\epsilon}} p=\tilde{\epsilon} \dot{\tilde{p}}, \quad \Delta_{\tilde{\epsilon}} \tilde{p}=\tilde{\epsilon} \dot{p}, & \Delta_{\tilde{\epsilon}} e=0=\Delta_{\tilde{\epsilon}} \tilde{e},
\end{array}
$$

is also a trivial symmetry. This shows that for diffeomorphism symmetry, the target space duality given by $J$ can be reabsorbed into a world-line duality $(e, \tilde{e}) \rightarrow(\tilde{e}, e)$. In this sense, the two canonical symmetries could be thought of as equivalent to 'chiral diffeomorphisms'. However, it is interesting to note that these chiral diffeomorphisms would be the separate world-line symmetries of independent 'chiral particles' that we referred to previously in Sec. III A. We saw there that this interpretation is impossible because it is impeded by both the $\alpha^{\prime}$ term in the symplectic potential as well as the causal choice of integration domain in $(e, \tilde{e})$. Indeed, the world-line duality $(e, \tilde{e}) \rightarrow(\tilde{e}, e)$ is not available in a restricted domain.

\section{INTERACTIONS AND BACKGROUNDS}

Finally, we would like to point to some issues involved in introducing interactions in the metaparticle theory. Here, we could have two things in mind. First, we might consider interactions that change particle number, corresponding to bifurcating world lines. Usually, this is side-stepped in favor of introducing a multi-particle field theory in target space. In the case of the metaparticle theory, what would such a field theory be? Perhaps it is a truncation of a string field theory in some sense (as we have discussed in $[13,14])$. We will leave discussion of this physics to future work, and point to a second notion of interactions, in which we couple the theory to background fields.

This structure is reminiscent of a similar procedure that would introduce a gauge field into the ordinary free particle theory. Indeed, let's review that here. Given an action

$$
S=\int d \tau\left(p \cdot \dot{x}-\frac{1}{2} e\left(p^{2}+m^{2}\right)\right)
$$

we may introduce a background gauge field by a shift of the symplectic potential

$$
S=\int d \tau\left(p \cdot \dot{x}+A(x) \cdot \dot{x}-\frac{1}{2} e\left(p^{2}+m^{2}\right)\right) .
$$

We notice that a gauge transformation $A_{\mu}(x) \mapsto A(x)-$ $\partial_{\mu} \varphi(x)$ has an interpretation as a canonical transformation with generating function $\varphi(x)$, under which the action changes by a total derivative. Denoting the canonical momentum by $P=p+A(x)$, we obtain

$$
S=\int d \tau\left(P \cdot \dot{x}-\frac{1}{2} e\left((P-A)^{2}+m^{2}\right)\right)
$$

which is the usual form of gauging in which the kinematic momentum appearing in the Hamiltonian is shifted to $P-A(x)$, with $P$ the canonical momenta (instead of a shift to the symplectic term).

Following this well-known procedure we might try to extend the gauging procedure to the metaparticle counterpart. There is a possible ambiguity in this gauging which depends on which configuration variables one decides to work with. If one take $(x, \tilde{x})$ as configuration variables, one obtains a gauging which could also be motivated by the presence of a "stringy gauge field" in metastring theory [3]

$$
\begin{aligned}
S \rightarrow & \int\left(\left(p_{\mu}+A_{\mu}(x, \tilde{x})\right) \dot{x}^{\mu}+\left(\tilde{p}^{\mu}+\tilde{A}^{\mu}(x, \tilde{x})\right) \dot{\tilde{x}}_{\mu}\right. \\
& \left.+2 \pi \alpha^{\prime} p_{\mu} \dot{\tilde{p}}^{\mu}-e \mathcal{H}(p, \tilde{p})-\tilde{e} \mathcal{D}(p, \tilde{p})\right) .
\end{aligned}
$$

Indeed, if we introduce canonical momenta

$$
P_{\mu}=p_{\mu}+A_{\mu}(x, \tilde{x}), \quad \tilde{P}^{\mu}=\tilde{p}^{\mu}+\tilde{A}^{\mu}(x, \tilde{x})
$$

we obtain then 


$$
\begin{aligned}
S \rightarrow & \int\left(P_{\mu} \dot{x}^{\mu}+\tilde{P}^{\mu} \dot{\tilde{x}}_{\mu}\right. \\
& +2 \pi \alpha^{\prime}\left(P_{\mu}-A_{\mu}(x, \tilde{x})\right)\left(\dot{\tilde{P}}^{\mu}-\frac{d}{d t} \tilde{A}^{\mu}(x, \tilde{x})\right) \\
& -e \mathcal{H}(P-A(x, \tilde{x}), \tilde{P}-\tilde{A}(x, \tilde{x})) \\
& -\tilde{e} \mathcal{D}(P-A(x, \tilde{x}), \tilde{P}-\tilde{A}(x, \tilde{x})))
\end{aligned}
$$

but, now, because of the $\alpha^{\prime}$ term we see that $\dot{\tilde{A}}$ contains $\dot{\tilde{x}}$.

Another way to proceed is to choose a Lagrangian subspace, say $(x, \tilde{p})$ and introduce a background which is form-valued on the Lagrangian. This would mean taking

$$
\begin{aligned}
S= & \int\left(\left(p_{\mu}+A_{\mu}(x, \tilde{p})\right) \dot{x}^{\mu}-\left(\tilde{x}_{\mu}+B_{\mu}(x, \tilde{p})\right) \dot{\tilde{p}}^{\mu}\right. \\
& \left.+2 \pi \alpha^{\prime} p_{\mu} \dot{\tilde{p}}^{\mu}-e \mathcal{H}(p, \tilde{p})-\tilde{e} \mathcal{D}(p, \tilde{p})\right) .
\end{aligned}
$$

As usual, this is gauge invariant under $A_{\mu} \mapsto$ $A_{\mu}+\frac{\partial}{\partial x^{\mu}} \Lambda(x, \tilde{p}), \quad B_{\mu} \mapsto B_{\mu}+\frac{\partial}{\partial \tilde{p}^{\mu}} \Lambda(x, \tilde{p})$, under which $S \mapsto S+\int d \tau \frac{d}{d \tau} \Lambda(x, \tilde{p})$. A change of variables $P=$ $p+A(x, \tilde{p})$ takes this to

$$
\begin{aligned}
S= & \int\left(P_{\mu} \dot{x}^{\mu}-\left(\tilde{x}_{\mu}+B_{\mu}(x, \tilde{p})+2 \pi \alpha^{\prime} A_{\mu}(x, \tilde{p})\right) \dot{\tilde{p}}^{\mu}\right. \\
& +2 \pi \alpha^{\prime} P_{\mu} \dot{\tilde{p}}^{\mu}-e \mathcal{H}(P-A(x, \tilde{p}), \tilde{p}) \\
& -\tilde{e} \mathcal{D}(P-A(x, \tilde{p}), \tilde{p})) .
\end{aligned}
$$

So we see that in fact it is convenient to introduce $\tilde{Q}=\tilde{x}+B+2 \pi \alpha^{\prime} A$

$$
\begin{aligned}
S= & \int\left(P_{\mu} \dot{x}^{\mu}-\tilde{Q}_{\mu} \dot{\tilde{p}}^{\mu}+2 \pi \alpha^{\prime} P_{\mu} \dot{\tilde{p}}^{\mu}\right. \\
& -e \mathcal{H}(P-A(x, \tilde{p}), \tilde{p})-\tilde{e} \mathcal{D}(P-A(x, \tilde{p}), \tilde{p}))
\end{aligned}
$$

with $\tilde{Q}$ acting as a Lagrange multiplier forcing $\tilde{p}$ to be constant along the world line. Notice the interesting result that $B_{\mu}$ has decoupled and the gauge field $A_{\mu}$ appears only in the Hamiltonian.

We will explore these different options and discuss more general interactions of metaparticles, as well as their physical interpretations, in a separate publication.

\section{CONCLUSION}

In this paper, we have begun an exploration of a new quantum particle model motivated by Born geometry in string theory. The model has been formulated as a worldline theory, in which a second local constraint, in addition to reparametrization invariance, is present. Although its origins in string theory are important, we regard the metaparticle as a new interesting theory in its own right. Indeed, one of our principle motivations is to study a theory that is Lorentz invariant in the presence of a fundamental length scale. It is natural to formulate such a theory in the context of Born geometry, in which the usual notion of spacetime (and, thus, locality) is not fixed, but appears as a subspace of a more general geometry that possesses specific properties. In the paper, we have carefully discussed properties of the theory alongside the more familiar relativistic free particle theory in order to bring out important concepts that are often lost in the usual gauge fixings employed for the latter. In particular, it is important to work in the full phase space of the theory.

Quantum transition amplitudes involve a choice of polarization of phase space. For the most part, we have confined our attention in this paper to the $x, \tilde{p}$ polarization, for which one might expect there to be a simple interpretation in terms of which $x$ coordinatizes a notion of spacetime. Indeed, one finds an interpretation of the transition amplitude as a particle propagator, with an extended pole structure, the details depending in particular on $\tilde{p}^{2}$. Despite this modification, the theory remains causal and unitary on the world line and possesses Lorentz symmetry although not in the standard manifest way. In addition, an important role is played by dualities, which play a role in the spectrum and propagation. The question of causality from a target spacetime point of view is not so obvious when $\tilde{p}$ is timelike. However, precisely in this case, the classical equations that relate the world-line frame variables and the target space coordinates do not have the usual classical particle gauge fixings, and so a classical interpretation of this case is not as straightforward as one might have expected. It is also in this context that we find, in addition to the antiparticle, a dual particle-antiparticle pair that stems from the presence of a fundamental scale.

To study the theory further, it would be natural to more closely examine the metaparticle theory reduced to the $x, \tilde{x}$ subspace of phase space. This is natural because these are local coordinates on (flat) Born geometry. However, this theory is noncommutative on the world line, and we have left its study to future work. Crucial to this study would be the introduction of interactions. One might envision this in several different ways. One path would be to formulate interactions in "first quantized" terms, by allowing world lines to bifurcate while preserving symmetries. This is the analogue of introducing $g_{\text {str }}$ in string theory, although the reader should be familiar with all the usual caveats of doing this in world-line theories. A second notion of interactions would appear in a "second quantized" field theory approach, although it is not readily apparent what this theory would entail. In the present paper, we have simply made some preliminary remarks in lieu of this by considering coupling the theory to (Abelian gauge) backgrounds. 
Further study of this may serve to clarify the physics of the pole structure of the metaparticle propagator.

\section{ACKNOWLEDGMENTS}

D. M. and J.K.G. thank the Perimeter Institute for hospitality. L.F., R. G. L. and D.M. thank the Julian Schwinger Foundation for support. D. M. also thanks the quantum gravity group of the Institute for Theoretical Physics at the University of Wroclaw for hospitality. R. G. L. is supported in part by the U.S. Department of Energy Contract No. DE-SC0015655 and D. M. by the U.S. Department of Energy under Contract No. DE-FG0213ER41917. For J. K. G., this work was supported by funds provided by the National Science Center, Project No. 2017/ 27/B/ST2/01902. Research at the Perimeter Institute for Theoretical Physics is supported in part by the Government of Canada through NSERC and by the Province of Ontario through MRI.

\section{APPENDIX: DISCRETIZATION AND ONE- DIMENSIONAL QUANTUM GRAVITY}

To understand how the world-line frame field enters the path integral (that is, how the $1 \mathrm{~d}$ diffeomorphism invariance is gauged), we provide details here, first for the ordinary particle, and then for the metaparticle. The analysis helps to understand the interplay between world-line causality and unitarity in the quantum theory.

For the ordinary particle, we usually consider the quantum transition amplitude between a state denoted $\left|x_{i}, \tau_{i}\right\rangle$ and a state $\left|x_{f}, \tau_{f}\right\rangle$,

$$
K\left(x_{f}, \tau_{f} ; x_{i}, \tau_{i}\right)=\left\langle x_{f}\left|e^{-i \hat{H}\left(\tau_{f}-\tau_{i}\right)}\right| x_{i}\right\rangle
$$

where $\tau$ is an intrinsic coordinate on a world-line segment $\tau \in\left(\tau_{i}, \tau_{f}\right)$ and the Hamiltonian is $H=p^{2}+m^{2}$. We discretize this line segment into $n$ subsegments labelled $\tau_{r}$ with $r=1,2, \ldots$ and use factorization to generate a path integral representation. It is standard to take these to be equally spaced, $\tau_{r+1}-\tau_{r}=\Delta \tau, \forall r$, where $\Delta \tau=\left(\tau_{f}-\tau_{i}\right) / n$ is a standard infinitesimal. However, it is a simple modification to allow an arbitrary spacing. To do so, we introduce

$$
\ell_{r+1}-\ell_{r}=e_{r} \Delta \tau
$$

We can regard this as the introduction of a chemical potential for the Hamiltonian $H$, and if we integrate over values of $e_{r}$, we will obtain reparametrization invariance in the continuum limit. The complication here, with implications for both causality in the target space and unitarity, is in deciding the measure of integration for the $e_{r}$. We write the transition amplitude now in the form
$K\left(x_{f}, \ell_{f} ; x_{i}, \ell_{i}\right)=\int \prod_{s=1}^{n-1} d^{d} x_{s} \prod_{r=0}^{n-1}\left\langle x_{r+1}\left|e^{-i \hat{H}(\hat{P})\left(\ell_{r+1}-\ell_{r}\right)}\right| x_{r}\right\rangle$.

In the present case, the Hamiltonian is a function of momenta only, $H=\mathcal{H}(p)=\frac{1}{2}\left(p^{2}+m^{2}\right)$, and so we can proceed by inserting complete sets of momentum eigenstates:

$$
\begin{aligned}
& K\left(x_{f}, \ell_{f} ; x_{i}, \ell_{i}\right) \\
& =\int \prod_{s=1}^{n-1} d^{d} x_{s} \prod_{s=0}^{n-1} \frac{d^{d} p_{s}}{(2 \pi)^{d}} \prod_{r=0}^{n-1} \int d e_{r}\left\langle x_{r+1}\left|e^{-i e_{r} \hat{H}\left(p_{r}\right) \Delta \tau}\right| p_{r}\right\rangle \\
& \quad \times\left\langle p_{r} \mid x_{r}\right\rangle \\
& \left.=\int \prod_{s=1}^{n-1} d^{d} x_{s} \prod_{s=0}^{n-1} \frac{d^{d} p_{s}}{(2 \pi)^{d}} \prod_{r=0}^{n-1} \int d e_{r} e^{i\left(p_{r}\left(x_{r+1}-x_{r}\right)-e_{r}\right.} \mathcal{H}\left(p_{r}\right) \Delta \tau\right) .
\end{aligned}
$$

In the continuum, we write this as

$$
\begin{aligned}
K\left(x_{f}, \ell_{f} ; x_{i}, \ell_{i}\right)= & \left.\int[d x(\tau)]\right|_{x_{i}} ^{x_{f}}[d p(\tau)][d e(\tau)] \\
& \times e^{i \int d \tau(p(\tau) \dot{x}(\tau)-e(\tau) \mathcal{H}(p(\tau)))} .
\end{aligned}
$$

Here, we have not specified yet the measure of integration over $e$, but we note that $e$ does appear, as expected, as a Lagrange multiplier, the gauge field of the world-line diffeomorphism invariance. In the quantum theory, what $K$ computes depends on the choice of integration measure for $e$. In fact, the "right answer" (or a right answer) is to take $e_{r} \geq 0, \forall r$. This choice is, in fact, consistent with factorization, ${ }^{14}$ and coincides with the discussion in the text in which we should think not in terms of a 1-form $e(\tau) d \tau$ but a density $|e(\tau)| d \tau$. For this choice, $K$ is the Feynman propagator, $(\mathcal{H}-i \epsilon)^{-1}$, the $i \epsilon$ being induced by regulating the integral over the zero mode of $e$, the external states being "off-shell" in that they are not annihilated by $\hat{H}$. Another choice consistent with unitarity is to take $e_{r} \in \mathbb{R}, \forall r$. For this choice, $K$ computes the "Hadamard propagator," $K \sim \delta[\mathcal{H}]$, and the external states are on-shell physical states. This is all related to causality in the target space because

\footnotetext{
${ }^{14}$ Since $e(\tau)$ is almost all pure gauge (e.g., a standard gauge fixing is $e(\tau)=\ell$, a constant) one might have thought that one could choose to integrate over all $e(\tau) \in \mathbb{R}$ with only the zero mode $\ell \geq 0$, but one can show that this choice violates unitarity. Note that here we are being somewhat cavalier with gauge fixing, but given that the $x$-integration forces $p$ to be trivial, only the zero mode $\ell=\int d \tau e(\tau)$ actually appears in the integrand. All other modes then are pure gauge and should be formally divided out. In the two cases discussed in the text, $\int[d e(\tau)] \rightarrow \int_{0}^{\infty} d \ell e^{-\ell \ell}$ (Feynman), versus $\int[d e(\tau)] \rightarrow \int_{-\infty}^{\infty} d \ell$ (Hadamard).
} 

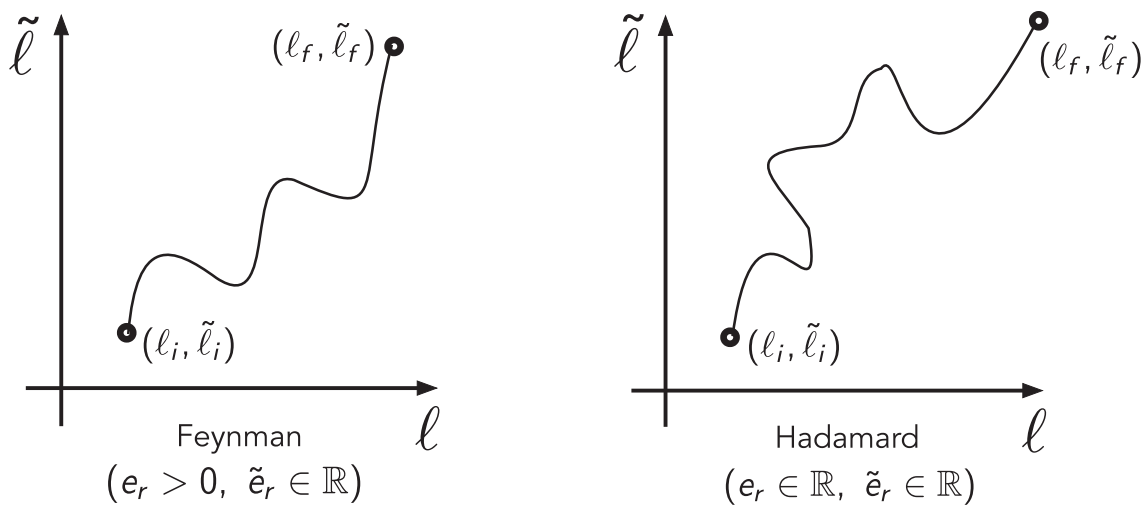

FIG. 1. The world line is discretized, with points labelled by $r$, each point on a world line mapping to $\left(\ell_{r}, \tilde{\ell}_{r}\right)$. In the continuum limit, we choose to take $r \rightarrow \tau, x_{r} \rightarrow x(\tau)$, etc. Typical paths, projected to $e, \tilde{e}$, are shown for the Feynman propagator and the Hadamard propagator, respectively.

canonically $\mathcal{H}$ generates the reparametrizations of the world line on the phase space variables.

For the metaparticle, there are a number of additional subtleties that arise. We know that since there are two constraints $\mathcal{H}$ and $\mathcal{D}$, there should be two Lagrange multipliers $e$ and $\tilde{e}$. It is of interest then to see how to generate these in a discretized world-line quantum theory, and to understand possible consistent choices of their integration measures. We will consider this construction in the $x, \tilde{p}$ polarization,

$$
\begin{aligned}
K\left(x_{f}, \tilde{p}_{f}, \ell_{f} ; x_{i}, \tilde{p}_{i}, \ell_{i}\right) & =\left\langle x_{f}, \tilde{p}_{f} ; \ell_{f} \mid x_{i}, \tilde{p}_{i} ; \ell_{i}\right\rangle \\
& =\left\langle x_{f}, \tilde{p}_{f}\left|e^{-i \hat{H}\left(\ell_{f}-\ell_{i}\right)}\right| x_{i}, \tilde{p}_{i}\right\rangle .
\end{aligned}
$$

Here, though, we have two constraints,

$$
\mathcal{H}=\left(p^{2}+\tilde{p}^{2}+m^{2}\right), \quad \mathcal{D}=(p \cdot \tilde{p}-\mu),
$$

and so a more general notion of quantum transition amplitude would include labels (a chemical potential) that conjugate to $\mathcal{D}$ as well

$$
\begin{aligned}
& K\left(x_{f}, \tilde{p}_{f}, \ell_{f}, \tilde{\ell}_{f} ; x_{i}, \tilde{p}_{i}, \ell_{i}, \tilde{\ell}_{i}\right) \\
& \quad=\left\langle x_{f}, \tilde{p}_{f} ; \ell_{f}, \tilde{\ell}_{f} \mid x_{i}, \tilde{p}_{i} ; \ell_{i}, \tilde{\ell}_{i}\right\rangle \\
& \quad=\left\langle x_{f}, \tilde{p}_{f}\left|e^{-i \hat{\mathcal{H}}\left(\ell_{f}-\ell_{i}\right)} e^{-i \hat{\mathcal{D}}\left(\tilde{\ell}_{f}-\tilde{\ell}_{i}\right)}\right| x_{i}, \tilde{p}_{i}\right\rangle .
\end{aligned}
$$

Note that $\ell_{f}-\ell_{i}>0$ by assumption, but the sign of $\tilde{\ell}_{f}-\tilde{\ell}_{i}$ is indeterminate. Discretization proceeds by introducing a proper time $\left\{\tau_{r}\right\}$ along the world line with $\Delta \tau=$ $\left(\tau_{f}-\tau_{i}\right) / n>0$ and supposing

$$
\ell_{r+1}-\ell_{r}=e_{r} \Delta \tau, \quad \tilde{\ell}_{r+1}-\tilde{\ell}_{r}=\tilde{e}_{r} \Delta \tau .
$$

This is an expression of the fact that we will obtain a onedimensional continuum limit. ${ }^{15}$ In the continuum limit, the wave-function factors give rise to the full symplectic form (accounting for the noncommutativity of $\hat{x}$ and $\hat{\tilde{x}}$ ), and we obtain

$$
\begin{aligned}
& K\left(x_{f}, \tilde{p}_{f}, \ell_{f}, \tilde{\ell}_{f} ; x_{i}, \tilde{p}_{i}, \ell_{i}, \tilde{\ell}_{i}\right) \\
& =\left.\int[d e(\tau) d \tilde{e}(\tau)] \int\left[d^{d} x(\tau) d^{d} \tilde{p}(\tau)\right]\right|_{x_{i}, \tilde{p}_{i}} ^{x_{f}, \tilde{p}_{f}} \int\left[d^{d} p(\tau) d^{d} \tilde{x}(\tau)\right] \\
& \quad \times e^{i \int d \tau\left(p \cdot \dot{x}-x \cdot \dot{\tilde{p}}+\pi \alpha^{\prime} p \cdot \dot{\tilde{p}}-e \mathcal{H}(p, \tilde{p})-\tilde{e} \mathcal{D}(p, \tilde{p})\right)} .
\end{aligned}
$$

We note that since

$$
\begin{array}{r}
\ell_{f}-\ell_{i}=\sum_{r}\left(\ell_{r+1}-\ell_{r}\right) \rightarrow \int d \tau e(\tau) \equiv \ell, \\
\tilde{\ell}_{f}-\tilde{\ell}_{i} \rightarrow \int d \tau \tilde{e}(\tau)=\tilde{\ell},
\end{array}
$$

we should regard this path integral as having a fixed value of $(\ell, \tilde{e})$. That is, the path integral over $[e(\tau), \tilde{e}(\tau)]$ is to be done with fixed zero mode. It is natural to average over values of $(\ell, \tilde{\ell})$ by integral transform. For example, we might consider integrating

$$
G=\int_{0}^{\infty} \mathrm{d} \ell f(\ell) \int_{-\infty}^{\infty} d \tilde{\ell} K(\ldots ; \ell, \tilde{\ell})
$$

with $f(x)=e^{-\epsilon x} \Theta(x)$ (Feynman, $\epsilon>0$ ) or $f(x)=1$ (Hadamard). It is one of these transition amplitudes averaged over $\tilde{\ell}$ that is employed in the text (See Fig. 1).

\footnotetext{
${ }^{15}$ Given the relation to string theory in which $\mathcal{D}$ generates world-sheet spatial translations, one might have thought that one could write instead $\tilde{\ell}_{r+1}-\tilde{\ell}_{r}=\tilde{e}_{r} \Delta \sigma$ and obtain a $1+1$ continuum limit, but since the theory does not contain all of the oscillator modes, we expect such a continuum limit does not exist.
} 
[1] L. Freidel, R. G. Leigh, and D. Minic, Born reciprocity in string theory and the Nature of spacetime, Phys. Lett. B 730, 302 (2014).

[2] L. Freidel, R. G. Leigh, and D. Minic, Quantum gravity, dynamical phase space and string theory, Int. J. Mod. Phys. D 23, 1442006 (2014).

[3] L. Freidel, R. G. Leigh, and D. Minic, Metastring theory and modular space-time, arXiv:1502.08005.

[4] L. Freidel, R. G. Leigh, and D. Minic, Modular spacetime and metastring theory, J. Phys. Conf. Ser. 804, 012032 (2017).

[5] G. Aldazabal, D. Marques, and C. Nunez, Double field theory: A pedagogical review, Classical Quantum Gravity 30, 163001 (2013).

[6] M. Gualtieri, Generalized complex geometry, arXiv: math/0401221.

[7] L. Freidel, F. J. Rudolph, and D. Svoboda, Generalised kinematics for double field theory, J. High Energy Phys. 11 (2017) 175.

[8] L. Freidel, F. J. Rudolph, and D. Svoboda, A unique connection for born geometry, arXiv:1806.05992.

[9] V. E. Marotta and R. J. Szabo, Para-Hermitian geometry, dualities and generalized flux backgrounds, arXiv: 1810.03953.

[10] G. Amelino-Camelia, L. Freidel, J. Kowalski-Glikman, and L. Smolin, The principle of relative locality, Phys. Rev. D 84, 084010 (2011).
[11] G. Amelino-Camelia, L. Freidel, J. Kowalski-Glikman, and L. Smolin, Relative locality: A deepening of the relativity principle, Gen. Relativ. Gravit. 43, 2547 (2011); Int. J. Mod. Phys. D 20, 2867 (2011).

[12] L. Freidel, R. G. Leigh, and D. Minic, Quantum spaces are modular, Phys. Rev. D 94, 104052 (2016).

[13] L. Freidel, R. G. Leigh, and D. Minic, Noncommutativity of closed string zero modes, Phys. Rev. D 96, 066003 (2017).

[14] L. Freidel, R. G. Leigh, and D. Minic, Intrinsic noncommutativity of closed string theory, J. High Energy Phys. 09 (2017) 060.

[15] J. Gomis and H. Ooguri, Nonrelativistic closed string theory, J. Math. Phys. (N.Y.) 42, 3127 (2001).

[16] E. C. G. Stueckelberg, Remarque à propos de la création de paires de particules en théorie de relativité, Helv. Phys. Acta 14, 51 (1941).

[17] T. Rempel and L. Freidel, Bilocal model for the relativistic spinning particle, Phys. Rev. D 95, 104014 (2017).

[18] T. Rempel and L. Freidel, Interaction vertex for classical spinning particles, Phys. Rev. D 94, 044011 (2016).

[19] S. Weinberg, The Quantum Theory of Fields. Vol. 1: Foundations (Cambridge University Press, Cambridge, England, 1995).

[20] J. J. Halliwell, Derivation of the Wheeler-De Witt equation from a path integral for minisuperspace models, Phys. Rev. D 38, 2468 (1988). 